\title{
Unusual increase of cases of myelitis in a pediatric hospital in Argentina
}

\author{
María Guadalupe Pérez, M.D. ${ }^{a}$, María T. Rosanova, M.D. ${ }^{a}$, María C. Freire, \\ Biochemist ${ }^{b}$, María I. Paz, M.D. ${ }^{c}$, Silvina Ruvinsky, M.D. ${ }^{a}$, Carlos Rugilo, M.D. ${ }^{d}$, \\ Victor Ruggieri, M.D. ${ }^{c}$, Daniel Cisterna, Biochemist ${ }^{b}$, Soledad Martiren, M.D. ${ }^{a}$, \\ Cristina Lema, Biochemist ${ }^{b}$, Andrea Savransky, M.D. ${ }^{c}$, Soledad González, M.D. ${ }^{a}$, \\ Leila Martínez, Biochemist ${ }^{b}$, Diana Viale, Biochemist ${ }^{e}$ and Rosa Bologna, M.D. ${ }^{a}$
}

\begin{abstract}
Introduction: The association between enterovirus D68 and acute flaccid myelitis was first described in the United States in 2014. Since then, sporadic cases have been reported in Canada and Europe. This study describes a series of cases of acute flaccid myelitis at Hospital de Pediatría "Prof. Dr. Juan P. Garrahan," in Buenos Aires, Argentina, during 2016.

Methods: Descriptive, retrospective study. All patients with acute flaccid myelitis and lesions in the spinal cord involving the gray matter, as observed in the magnetic resonance imaging (MRI) scan, hospitalized from $04 / 01 / 2016$ to $07 / 01 / 2016$, wereincluded in the study. Samples of nasopharyngeal secretions, fecal swabs and cerebrospinal fluid were collected and processed to look for the causative agent.

Results: Ten patients were included. The median age was 4 years old (range from 3 months to 5 years old). Eight patients had a self-limiting febrile condition before the onset of neurological symptoms. Neurological findings were flaccid weakness in, at least, one limb, cervical paralysis $(\mathrm{n}=2)$ and facial paralysis $(\mathrm{n}=2)$. All patients had longitudinal lesions in the spinal cord, with gray matter involvement, mainly in the anterior horn. In all cases, a lumbar puncture (spinal tap) was performed. Pleocytosis was observed in 7 patients. In four children, enterovirus D68 was identified in nasopharyngeal secretions, and in one, it was detected in the cerebrospinal fluid. Neurological deficit persisted in all patients at the time of discharge.

Conclusions: The first outbreak of acute flaccid myelitis associated to enterovirus D68 is reported in Argentina. Active epidemiological surveillance will help to determine the true incidence, epidemiology and etiology of this disease.

Key words: acute flaccid myelitis, enterovirus, poliomyelitis, pediatrics, human enterovirus D68.
\end{abstract}

http:/ / dx.doi.org/10.5546/ aap.2017.eng.364

E-mail address:

María G. Pérez, M.D.:

guaperez@hotmail.com

Funding: None.

Conflict of interest:

None.

Received: 9-17-2016

Accepted: 1-25-2017 To cite: Pérez MG, Rosanova MT, Freire MC, et al. Unusual increase of cases of myelitis in a pediatric hospital in Argentina. Arch Argent Pediatr 2017;115(4):364-369.

\section{INTRODUCTION}

The introduction of polio vaccines in the 1950s helped to reduce the number of cases of acute flaccid paralysis associated to poliovirus infection worldwide. In the last decades, there have been reports of cases of acute flaccid paralysis with a variable degree of gray matter involvement, associated to other nonpolio enteroviruses (enterovirus A71, D68, Coxsackievirus A and B) and other neurotropic viruses (flavivirus, herpes virus, adenovirus), among others. ${ }^{1}$

In 2014, in the United States, an increase in the number of cases of acute flaccid paralysis was reported, associated to the identification of enterovirus D68 (EVD68) in nasopharyngeal secretions (NPS) after an outbreak of severe respiratory symptoms associated with this virus. Most of these patients had unusual MRI findings in the spinal cord which were defined as acute myelitis to differentiate them from other manifestations of flaccid paralysis. ${ }^{2}$

In Argentina, the surveillance program for acute flaccid paralysis, which includes all cases occurred in children younger than 15 years old, reported 204 cases in 2015. ${ }^{3}$ At Hospital de Pediatría "Prof. Dr. Juan P. Garrahan", in that same year, 15 children with acute flaccid paralysis were studied.

As from the first week of April, 2016, a remarkably high number of cases of children with acute flaccid paralysis was reported in this hospital. The condition was characterized by flaccid paralysis, absence of deep 
tendon reflexes in one or more limbs, no alteration of consciousness and an MRI scan showing signs of myelitis with involvement of the anterior horn.

Given the alarming increase of these manifestations compatible with acute myelitis, a diagnosis and follow-up protocol was developed to analyze the clinical, epidemiological and evolutionary characteristics in these patients so as to promote the epidemiological surveillance and implement timely actions, when relevant.

\section{OBJECTIVES}

To describe the incidence as well as the clinical, epidemiological, etiological and evolutionary characteristics of this condition.

\section{MATERIAL AND METHODS}

A descriptive, observational study was conducted.

Patients hospitalized at Hospital de Pediatría "Prof. Dr. Juan P. Garrahan" with the diagnosis of acute flaccid paralysis and myelitis were registered.

Study period: from 04/01 / 2016 to 07 / 01 / 2016.

Inclusion criteria: asymmetrical flaccid paralysis of sudden onset, involving one or more limbs, absence of deep tendon reflexes in one or more limbs, MRI showing signs of longitudinal myelitis with involvement of the gray matter of the anterior horn.

Exclusion criteria: history of trauma, electromyography compatible with polyradiculoneuropathy (or Guillain-Barre Syndrome), sensory involvement, MRI scan showing bone or intervertebral disk involvement.

Ancillary tests: all patients underwent cerebrospinal fluid (CSF) testing, cytochemical examination and culture to detect and identify common microorganisms, mycobacteria and fungi. Samples were also analyzed using polymerase chain reaction (PCR) to detect enteroviruses, herpes viruses (herpes simplex 1-2, 6, Epstein-Barr virus, cytomegalovirus, varicella zoster) and Mycoplasma pneumonia bacteria.

Respiratory viruses (adenovirus, influenza A, influenza B) were studied in NPS with immunofluorescence technique. PCR was also performed for adenovirus, influenza, enterovirus and Mycoplasma pneumoniae identification. Fecal specimens were submitted to the Department of Virus-induced Neurological Diseases of the National Infectious Disease Institute (Instituto Nacional de Enfermedades Infecciosas, INEI)-
National Administration of Health Institutes and Labs (Administración Nacional de Laboratorios e Institutos de Salud, ANLIS) "Dr. Carlos G. Malbrán" to be tested for enteroviruses (non-polio enterovirus, poliovirus, Sabin, Sabin-derived), in compliance with the acute flaccid paralysis protocol.

Epstein-Barr virus, cytomegalovirus, varicella virus, human immunodeficiency virus (HIV), arboviruses (San Luis, West Nile virus, Zika and dengue) and mycoplasma spp. serology was also used to identify likely etiologies.

At the Department of Virus-induced Neurological Diseases, samples were tested to detect and identify human enteroviruses/human rhinoviruses (HEV/HRV). Viruses detected in any of the samples were identified using a nested generic RT-PCR and subsequent genome sequencing.

Imaging examinations: An MRI scan was performed in all patients to assess the spinal cord and the brain.

Follow-up and assessment: Patients were evaluated by the Departments of Neurology, Internal Medicine and Infectious Diseases from their first day of hospitalization up to, at least, 30 days after discharge.

All examinations were part of the standard medical care for this condition and clinical course of patients. Data analysis and publication were not disclosed, protecting patients and their families' identity.

\section{RESULTS}

During the study period, 10 patients who met the inclusion criteria were identified. Their median age was 4 years old (range: 3 months to 5 years old); 6 were male. All children were immunocompetent; one patient had an associated comorbidity (polymicrogyria, without clinical manifestations until the onset of myelitis. A previous MRI scan showed no abnormalities in the medulla, pons nor spinal cord).

Patients were from several districts of the Province of Buenos Aires and from the Autonomous City of Buenos Aires. No cases were interrelated.

Most children (n: 8, 80\%) had a history of respiratory infection within 10 days prior to the onset of the paralysis.

All of them had flaccid paralysis in any of the limbs (upper: 8, lower: 2). Cervical paralysis was demonstrated in 2 patients, and in other 2 patients facial paralysis was confirmed when they were 
concomitantly admitted to the hospital. Nine patients had fever at the time of admission.

All children underwent lumbar puncture. Cytochemical testing of CSF revealed pleocytosis in 7 out of the 10 patients $(70 \%)$, with a median of 32-element cells / $\mathrm{mL}$ (range: 7-187). In all cases, lymphocytes were predominant.

An electromyography was performed in 6 patients, showing that peripheral nerves were unaffected in all of them.

MRI scans of all children showed abnormalities on T2 and FLAIR images, which did not enhance with the administration of IV contrast, with central spinal cord or anterior horn involvement, and spinal cord swelling (Figures 1 and 2). Lesions extended longitudinally in a variable caudal fashion. Medulla and pons involvement was documented in 4 patients.

Microbiological results are shown on Table 1. EVD68 was identified in 4 patients; in 3 of them in NPS samples, and in one both in NPS and CSF samples. In the remaining samples, other enteroviruses, usually present in NPS and feces in our setting, were identified.

The following treatments were given: methylprednisolone pulses (n: 9), IV gamma

FIGURE 1. MRI scan of the spinal cord. Cervical spinal cord swelling is demonstrated in one case

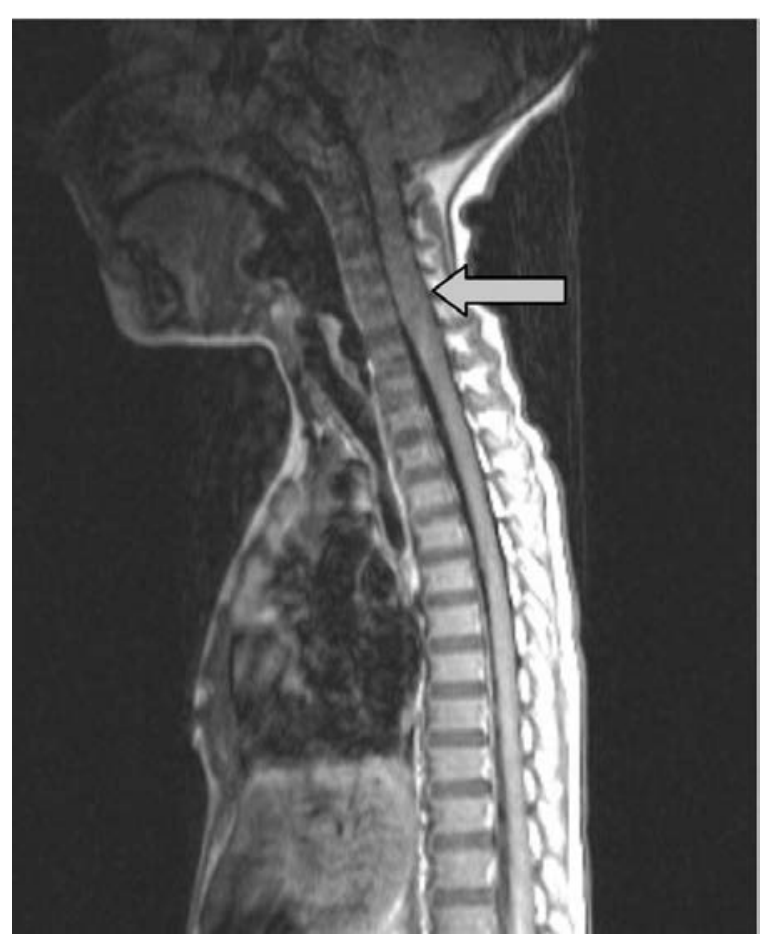

globulin (n: 5) and plasmapheresis in 3 patients.

Four patients experienced worsening of their clinical condition with progression of weakness, and admission to the ICU was required.

The median length of stay at the hospital was 26 days (range: $12-116$ ). During the 30 -day followup, two of the patients continued with assisted mechanical ventilation and 4 patients showed a mild improvement, with increased head control. However, all children experienced residual paresis in the affected limbs. There were no deaths. At the time of this publication, all patients were still being followed-up at the hospital.

\section{DISCUSSION}

The incidence of acute flaccid paralysis decreased dramatically worldwide since the development and universal use of polio vaccines. In Argentina, the latest case of poliomyelitis was recorded in 1984. In 1994, the World Health Organization (WHO) declared America a polio-free continent. ${ }^{3}$ Since the eradication of

FIGURE 2. MRI scan. Enhancement in the anterior horn region of the cervical spinal cord in one of the cases (arrow)

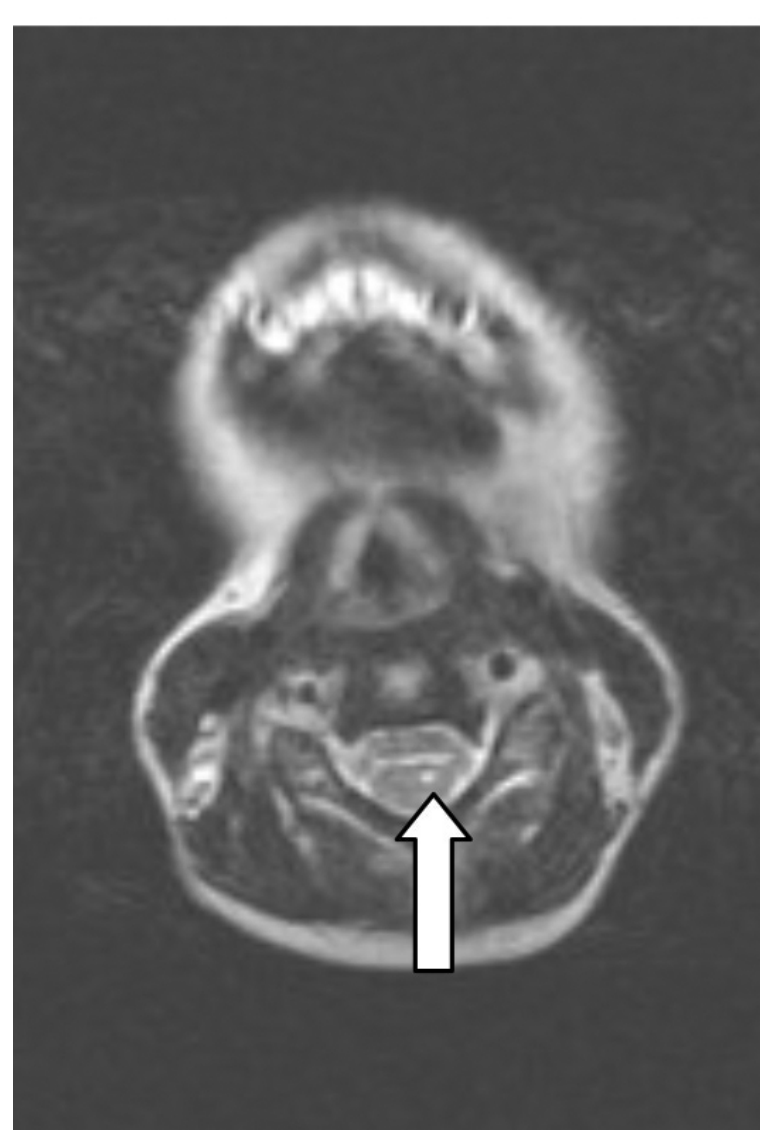


poliomyelitis, other non-polio enteroviruses -associated to acute flaccid paralysis and with the potential to cause small outbreaks- became relevant..$^{1-5}$ In this condition, myelitis is rare and can be secondary to infectious and non-infectious causes, which should be carefully assessed. The main infectious causes are those related to enteroviruses (polio and non-polio), arboviruses (dengue, Zika, Chikungunya and West Nile virus), adenoviruses and bacteria, such as Lyme disease or Rickettsiosis, among others. ${ }^{4}$

The unusual number of myelitis cases seen at our Hospital, registered in a very short period of time and without any epidemiological link among them, as well as the similarity of the clinical presentation in patients, shows consistency with cases reported in the United States and Europe. ${ }^{5,6}$ In the United States, 120 patients with confirmed myelitis were registered in 2014. Their median age was 7 years and most of them were male. ${ }^{7}$

Similar to data published by the Centers for Disease Control and Prevention (CDC), ${ }^{3}$ in this series, most patients had had a history of febrile disease with respiratory symptoms during the week preceding the onset of neurological symptoms, with a sudden onset of weakness in a limb, gray matter involvement in the MRI scan and lack of response to treatment, as well as persistence of the neurological deficit.

Limb weakness, which was the most frequent clinical manifestation, was consistent with the cases described in literature. ${ }^{4}$

In agreement with our findings, pleocytosis was present in $74 \%$ of patients reported in the United States. ${ }^{7}$

In the outbreak reported in the United States, EVD68 was identified in the NPS of $20 \%$ of patients studied and in $47 \%$ when samples were submitted before 7 days after the onset of symptoms. ${ }^{4}$

As from the identification of the increase of myelitis cases at the Hospital, the screening for etiological agents in NPS was implemented. In $40 \%$ of the children in this series, EVD68 was identified in respiratory secretions, probably due to early sample collection. The identification in NPS and feces of different enteroviruses commonly found in our setting, with intermittent viral shedding after infections, would be a finding. Therefore, a reliable causal association with the described clinical manifestations cannot be established at present.

Among cases reported in the literature, EVD68 was identified in the CSF of only one patient;

TABLE 1. Characteristics of the cerebrospinal fluid, virological and radiological findings, and Intensive Care Unit requirements

\begin{tabular}{|c|c|c|c|c|c|c|c|c|}
\hline \multirow[t]{2}{*}{ Case } & \multicolumn{3}{|c|}{$\begin{array}{c}\text { Cytochemistry } \\
\text { CSF } \\
\end{array}$} & \multirow{2}{*}{$\begin{array}{l}\text { Nasopharyngeal } \\
\text { secretions }\end{array}$} & \multirow[t]{2}{*}{ Feces } & \multirow[t]{2}{*}{ CSF } & \multirow[t]{2}{*}{$\begin{array}{l}\text { Location of pathological images } \\
\text { in the MRI scan }\end{array}$} & \multirow[t]{2}{*}{$\begin{array}{l}\text { Requires } \\
\text { ICU }\end{array}$} \\
\hline & $\begin{array}{l}\text { Glucose } \\
\mathrm{mg} / \mathrm{dL}\end{array}$ & $\begin{array}{l}\text { Proteins } \\
\mathrm{mg} / \mathrm{dL}\end{array}$ & $\begin{array}{c}\text { Cells } \\
\text { (in } \mathrm{mm}^{3} \text { ) }\end{array}$ & & & & & \\
\hline 1 & 67 & 107 & 187 & EVD68 & Enterovirus B & EVD68 & $\begin{array}{l}\text { Medulla, pons and anterior horn } \\
\text { at C3-C6 and T11-L1 }\end{array}$ & Yes \\
\hline 2 & 62 & 37 & 10 & EVD68 & Enterovirus C & & Medulla, pons and cervical spinal cord & Yes \\
\hline 3 & 56 & 32 & 3 & EVD68 & & & Pons and cervical myelitis & No \\
\hline 4 & 77 & 46 & 32 & EVD68 & & & $\begin{array}{l}\text { Medulla, pons and cervical spinal cord } \\
\text { up to the terminal cone }\end{array}$ & No \\
\hline 5 & 77 & 45 & 32 & & Enterovirus C & & C2 Cervical spinal cord to L1 & Yes \\
\hline 6 & 56 & 36 & 38 & Enterovirus B & Enterovirus B & & $\begin{array}{l}\text { Cervical and thoracic spinal cord } \\
\text { up to the terminal cone }\end{array}$ & No \\
\hline 7 & 69 & 37 & 0 & $\begin{array}{l}\text { ECHO Virus } \\
\text { Adenovirus }\end{array}$ & Enterovirus C & & $\begin{array}{l}\text { Central terminal cone involvement } \\
\text { Punctate signal pattern in the } \\
\text { anterolateral region of right C4-C5 }\end{array}$ & Yes \\
\hline 8 & 46 & 38 & 7 & Rhinovirus C & & & Central thoracic spinal cord involvement & No \\
\hline 9 & 63 & 29 & 0 & Enterovirus A & & & $\begin{array}{l}\text { Medulla, pons and anterior horns of } \\
\text { cervical spinal cord C3-C6 }\end{array}$ & No \\
\hline 10 & 58 & 31 & 24 & Influenza A & & & C2-C7 Cervical spinal cord & No \\
\hline
\end{tabular}

EVD68: enterovirus D68; ECHO: enteric cytopathic human orphan; CSF: cerebrospinal fluid; MRI: magnetic resonance imaging; ICU: intensive care unit. C: cervical; T: thoracic; L: lumbar. 
however, the authors reported that the material was contaminated with the patient's blood, and they suggested that the finding was probably due to such contamination. ${ }^{2}$ In this study, the finding of EVD68 in the CSF of one of the patients is striking. It could be attributed to early sample collection.

The EVD68, which was first described in 1962, belongs to the Picornaviridae family. Viruses in this family generally infect the gastrointestinal and respiratory tracts, and cause mild symptoms. Nevertheless, they may invade the central nervous system and cause different degrees of infection, which in some cases are associated to high morbidity and mortality. The physiopathogenic mechanism of these viruses is unknown. ${ }^{8}$ Direct nervous tissue cytotoxicity and other mechanisms related to an inflammatory response have been postulated. ${ }^{9}$

Until 2014, cases reported of EVD68 were scarce worldwide. However, as of 2014, an increasing number of patients hospitalized with serious respiratory infections and EVD68 in NPS has been registered in the United States. ${ }^{2}$ At the same time, admissions due to respiratory infections with the identification of this same virus were reported in Canada, Europe and Asia. ${ }^{10}$ Some of these patients had flaccid paralysis, and myelitis was identified in their MRI scans. ${ }^{11}$

It is worth mentioning, that the main MRI characteristics are the selective involvement of the gray matter, with hyperintense lesions on T2 and FLAIR images that are not enhanced with IV contrast. A typical " $\mathrm{H}$ " pattern is observed due to the involvement of the anterior horn. No abnormalities are seen in the anterior or posterior roots. Spinal cord involvement can be observed at different levels (from the cervical to the thoracic level), but its extension is longitudinal. The terminal cone and the cauda equina are frequently affected. ${ }^{12}$

In the series presented, MRI findings are identical to those reported in the literature.

The lack of response to treatment is consistently described in the literature.

In has not been demonstrated that the specific antiviral treatment improves the prognosis in these patients. ${ }^{13}$ Some studies conducted with pleconaril for the treatment of neonatal sepsis due to enteroviruses showed a shorter viral shedding duration, but no clinical improvement. ${ }^{14}$

In vitro studies suggest that fluoxetine might decrease RNA synthesis, but it cannot modulate the entry of viruses into cells. Itraconazol, mefloquine and nitazoxanide have also been studied. ${ }^{15}$ None of these treatment strategies has been assessed in humans and their true clinical usefulness is unknown. ${ }^{16}$ Therefore, they were not used in these patients.

The immunomodulatory treatment has not shown any benefits in other countries where patients with these clinical characteristics were assisted. The use of corticosteroids, gamma globulin and plasmapheresis is generally not recommended. ${ }^{17}$

In most of the cases reported, disease progression is not free of sequelae. ${ }^{7}$ Some investigators consider that EVD68 could be a necessary but not sufficient factor for the development of acute flaccid myelitis. An autoimmune mechanism might also be involved.

Considering that EVD68 was isolated in 40\% of patients, it is necessary to monitor and identify enteroviruses that are frequent in our setting, in order to assess the true magnitude of the risk of myelitis associated to these emerging viruses.

A warning is issued about the presence of this unusual number of cases of myelitis in this pediatric center, so that a thorough investigation of its etiology is conducted. Early sample collection could favor the etiologic diagnosis. Cases should be immediately reported and microbiological tests carried out at once so as to rule out infections by polio viruses and derivatives.

An early identification of cases and a thorough examination of patients are critical to establish the causal association between this condition and EVD68. An active epidemiological surveillance and a multidisciplinary approach are required to determine the clinical, virological and evolutionary characteristics of this disease like acute myelitis with gray matter involvement, which causes a high degree of morbidity in affected children.

\section{CONCLUSIONS}

The first outbreak of acute flaccid myelitis associated to EVD68 is reported in Argentina. An active epidemiological surveillance will reveal the actual incidence, epidemiology and etiology of this disease.

\section{REFERENCES}

1. Israele V. Encefalomielitis. En Paganini H. Infectología pediátrica. Buenos Aires: Interamericana; 2007.P.529-38.

2. Greninger AL, Naccache SN, Messacar K, et al. A novel outbreak enterovirus D68 strain associated with acute flaccid myelitis cases in the USA (2012-14): a retrospective 
cohort study. Lancet Infect Dis 2015;15(6):671-82.

3. Organización Mundial dela Salud. Vigilancia dela parálisis flácida aguda en las Américas. Boletín Semanal de Polio 2016;31(2). [Accessed on: September 2016]. Available at: http:/ / www.paho.org/hq/index. php ?op t ion=com cont ent \&view=art icle\&id=295\% 3A2008-polio-weeklybulletin\&catid $=4049 \% 3$ Apolio-publications\&Itemid $=362$ 6\&lang $=\mathrm{es}$.

4. Macesic N, Hall V, Mahony A, et al. Acute Flaccid Paralysis: The New, The Old, and The Preventable. Open Forum Infect Dis 2015;3(1):ofv190.

5. Messacar K, Schreiner TL, Van Haren K, et al. Acute flaccid myelitis: A clinical review of US cases 2012-2015. Ann Neurol 2016;80(3):326-38.

6. Calvo C, Rodrigo C, Cabrerizo M, et al. Infecciones por enterovirus. Revisión de la situación en España. Sociedad Española de Infectología Pediátrica. [Accessed on: September 2016]. Available at: http:/ / www.aeped.es/ sites/default / files/documentos/enterovirusseip.pdf.

7. SejvarJJ, Lopez AS, Cortese MM, etal. Acute Flaccid Myelitis in the United States, August-December 2014: Results of Nationwide Surveillance. Clin Infect Dis 2016;63(6):737-45.

8. Huang HI, Shih SR. Neurotropic Enterovirus Infections in the Central Nervous System. Viruses 2015;7(11):6051-66.

9. Messacar K, Abzug MJ, Dominguez SR. 2014 outbreak of enterovirus D68 in North America. J Med Virol 2016;88(5):739-45.
10. Holm-Hansen CC, Midgley SE, Fischer TK. Global emergence of enterovirus D68: a systematic review. Lancet Infect Dis 2016:16(5):e64-75.

11. Messacar K, Schreiner TL, Maloney JA, et al. A cluster of acute flaccid paralysis and cranial nerve dysfunction temporally associated with an outbreak of enterovirus D68 in children in Colorado, USA. Lancet 2015;385(9978):166271.

12. Maloney JA, Mirsky DM, Messacar K, et al. MRI findings in children with acute flaccid paralysis and cranial nerve dysfunction occurring during the 2014 enterovirus D68 outbreak. AJNR Am J Neuroradiol 2015;36(2):245-50.

13. Abzug MJ. The enteroviruses: problems in need of treatments. J Infect 2014;68(Suppl 1):S108-14.

14. Abzug MJ, Cloud G, Bradley J, et al. Double blind placebocontrolled trial of pleconaril in infants with enterovirus meningitis. Pediatr Infect Dis J 2003;22(4):335-41.

15. Tyler KL. Rationale for the evaluation of fluoxetine in the treatment of enterovirus D68-associated acute flaccid myelitis. JAMA Neurol 2015;72(5):493-4.

16. Esposito S, Bosis S, Niesters H, et al. Enterovirus D68 Infection. Viruses 2015;7(11):6043-50.

17. Gerber S, Jackson MA, Johnson D. Enterovirus D68 in the United States: Epidemiology, Diagnosis \& Treatment. Center of diseases control and prevention; 2014. [Accessed on: July 2016]. Available at: https:/ / emergency.cdc.gov/ coca/calls/2014/callinfo_091614.asp. 